\title{
Protective effect of Qishen Yiqi dropping pills on the myocardium of rats with chronic heart failure
}

\author{
YINGHONG LU*, DAN WANG* ${ }^{*}$, XIAOLI YUAN, MENGCHAO WANG, \\ ZHIHUI LI, XIAOQING BAO, CHENGJIA ZHANG and HAIYUN JING \\ Department of Cardiology (V), Zhengzhou Central Hospital Affiliated to \\ Zhengzhou University, Zhengzhou, Henan 450007, P.R. China
}

Received April 9, 2018; Accepted October 18, 2018

DOI: $10.3892 /$ etm.2018.6957

\begin{abstract}
Protective effect of Qishen Yiqi dropping pills on the myocardium of rats with chronic heart failure (CHF) was investigated. Sixty rats were divided into the sham operation $(n=20)$, the model $(n=20)$ and the Qishen Yiqi dropping pill treatment group $(n=20)$ using the random table method. The treatment group received administration of Qishen Yiqi dropping pills. The model and the sham operation group were given the same amount of normal saline. Within $24 \mathrm{~h}$ after the last administration, the rats were sacrificed. The myocardia were used for reverse transcription-polymerase chain reaction, western blot analysis and histological examination. In the sham operation group, cardiomyocytes were stained evenly and arranged neatly and densely with clear structures. In the model group, the cell morphology was fuzzy, the myocytes were hypertrophied, the nuclear pyknosis was fragmented, the arrangement was disordered, the intercellular space was narrowed, and the cytoplasm was missing. The apoptosis rates of cardiomyocytes in the model and Qishen Yiqi dropping pill treatment group were significantly higher than that in the sham operation group $(\mathrm{P}<0.05)$. The myocardial infarction areas in the model group and the Qishen Yiqi dropping pill treatment group were larger than that in the sham operation group $(\mathrm{P}<0.05)$. The expression levels of transforming growth factor- $\beta 1$, mothers against decapentaplegic homolog 2 (Smad2), Smad3, and caspase-3 messenger ribonucleic acids and proteins in the model group and the Qishen Yiqi dropping pill treatment group were higher than those in the sham operation group $(\mathrm{P}<0.05)$. Qishen Yiqi dropping pills have
\end{abstract}

Correspondence to: Dr Haiyun Jing, Department of Cardiology (V), Zhengzhou Central Hospital Affiliated to Zhengzhou University, 195 Tongbai Road, Zhengzhou, Henan 450007, P.R. China

E-mail: jg28eg@163.com

${ }^{*}$ Contributed equally

Key words: chronic heart failure, Qishen Yiqi dropping pills, myocardial fibrosis, apoptosis an obvious myocardial protective effect on CHF rats, which may enhance the degree of myocardial fibrosis by inhibiting the TGF- $\beta 1 /$ Smads pathway and improve cardiomyocyte apoptosis by suppressing the caspase- 3 signaling pathway, thus protecting the myocardium.

\section{Introduction}

Chronic heart failure (CHF) is one of the manifestations and the main causes of death of various cardiovascular diseases in the terminal stage (1). Prevention and treatment for $\mathrm{CHF}$ in coronary heart disease are one of the impotant research issues world-wide. The pathogenesis of CHF is very complicated, and it is considered that the cardiac overload, mainly characterized by pathological remodeling of the left ventricle, involving various factors and primarily manifested as neuroendocrinology, is the main cause of CHF (2). Traditional Chinese medicine has certain advantages in the treatment of $\mathrm{CHF}$, such as minor side effects, improvement of symptoms, whole recuperative medical care and improvement of the patient's quality of life. The main components of Qishen Yiqi dropping pills are Danshen (Radix Salviae Miltiorrhizae), Huangqi (radix astragali), Sanqi (radix notoginseng) and Jiangxiang oil (Lignum Dalbergiae Odoriferae Oil), and the pills have effects of activating blood circulation, relieving pain, and promoting circulation of qi. Qishen Yiqi dropping pills have been proved to have certain curative effects in the treatment of CHF with few adverse reactions (3). However, there is still no report on the exact mechanism of CHF treatment with Qishen Yiqi dropping pills. This study aims to analyze the protective effect of Qishen Yiqi dropping pills on the myocardium of rats by establishing the rat model of CHF with Sprague-Dawley (SD) rats as subjects.

\section{Materials and methods}

Experimental animals and grouping. A total of 60 specificpathogen-free (SPF) SD male rats weighing 250-360 g, with an average weight of $280 \pm 15 \mathrm{~g}$ were purchased from Shanghai SLAC Laboratory Animal Center Co., Ltd (Shanghai, China). The rats were fed to acclimate for 1 week. They were kept in cages in the dark at $25^{\circ} \mathrm{C}$ with enough millet and cold boiled water. A total of 60 rats were randomly divided into the sham 
operation $(n=20)$, the model $(n=20)$ and Qishen Yiqi dropping pill treatment $(n=20)$ groups.

The study was approved by the Ethics Committee of Zhengzhou Central Hospital Affiliated to Zhengzhou University (Zhengzhou, China).

Main reagents. Qishen Yiqi dropping pills (Tasly Pharmaceutical Group Co., Ltd., Shanghai, China; NMPN Z20030139), hydrated chloral mixture (Sigma-Aldrich; Merck KGaA, Darmstadt, Germany) and terminal deoxynucleotidyl transferase dUTP nick end labeling (TUNEL) detection kit (Shanghai Ming Bo Biological Technology Co., Ltd., Shanghai, China) were used in the study. Other reagents were analytically pure and made in China.

Main instruments. Instruments used in the study were: Clean bench (Suzhou Purification Equipment Co., Ltd., Suzhou, China), desk-type cryogenic high-speed refrigerated centrifuge (Taicang Medical Appliance Factory, Jiangsu, China), ultraviolet spectrophotometer and electrophoresis apparatus (Shanghai No. 3 Analytical Instrument Factory, Shanghai, China), BS223S type electronic balance (Sartorius Scientific Instruments Co., Ltd., Beijing, China), and iChem530 automatic biochemical analyzer (Shenzhen ICUBIO Biotechnology Co., Ltd., Shenzhen, China).

Establishment of the rat model of CHF. The CHF model was established via coronary artery ligation. Hydrated chloral mixture was intraperitoneally injected to anaesthetize the potruncus of rats for skin preparation, rats were connected to small animal ventilator. The skin at the pulsating cardiac apex was cut to expose the heart, and the anterior descending coronary artery was cut with a 0 -gauge suture. The myocardial ischemic changes for $30 \mathrm{~min}$ displayed in an electrocardiogram indicated a successful surgery. After the model was successfully established, the heart was quickly laid back, and the chest wall was sutured. Intramuscular injection of penicillin was conducted to prevent infection for 7 consecutive days. In the sham operation group, the left anterior descending coronary artery was only occluded with sutures but not ligated after thoracotomy. The other procedures were the same as above.

Administration methods. On the 7th day after modeling, the treatment group was intragastrically administered with Qishen Yiqi dropping pills $(0.135 \mathrm{~g} / \mathrm{kg})$ once a day for 4 consecutive weeks. The model and the sham operation groups were given the same amount of normal saline.

\section{Observational indexes}

Material drawing. Within $24 \mathrm{~h}$ after the last administration, the rats were sacrificed, the heart was isolated, the bilateral atria and right ventricle were removed, the left ventricle was taken, and some of the fresh left ventricular myocardia were taken. Part of the myocardia were applied for reverse transcription-polymerase chain reaction (RT-PCR) and western blot analysis, and part of the left ventricle tissues were fixed with $4 \%$ paraformaldehyde for histological examination.

Histopathological detection. Myocardial tissues were taken, routinely embedded in paraffin and sectioned. In a staining jar, paraffin sections were washed with xylene and eluted with gradient alcohol. Hematoxylin and eosin (H\&E) staining was performed, followed by observation under an optical microscope (Shanghai Yongke Optical Instrument Co., Ltd., Shanghai, China).

Detection of cell apoptosis via TUNEL. Myocardial tissues were taken and routinely embedded in paraffin and sectioned. In a staining jar, paraffin sections were rinsed in xylene and eluted by using gradient alcohol. The tissues were treated with proteinase K for 15-30 min and washed with phosphate-buffered saline (PBS), followed by addition of TUNEL reaction mixture. After the glass slide was air-dried, 50 $\mu 1$ TUNEL reaction mixtures were added to samples for reaction in a wet box at $37^{\circ} \mathrm{C}$ for $1 \mathrm{~h}$, followed by rinsing with PBS three times. Then 50-100 $\mu 1$ diaminobenzidine (DAB) substrates were added for reaction at $15-25^{\circ} \mathrm{C}$ for $10 \mathrm{~min}$, followed by washing with PBS three times. Subsequently, the tissues were counterstained with hematoxylin, and dehydrated with gradient alcohol and made transparent with xylene, followed by mounting using neutral gums. Apoptotic cells (200-500 cells in total) were observed under an optical microscope. The apoptosis index was calculated according to apoptosis index $=$ the number of apoptotic cells x 100\%/total cell number.

Detection of myocardial infarction area. Heart samples were taken and immediately frozen in liquid nitrogen. The samples were continuously sectioned along the long axis of the heart from the ligation point to the cardiac apex and stained with triphenyltetrazolium chloride (TTC). Through observation by naked eye, normal tissues were brick-red, and tissues in the ischemic area were gray-white. Infarction area was calculated by using image processing software. Infarction area $=$ ischemic area/total area of the left ventricle $\mathrm{x} 100 \%$.

Detection of the expression levels of transforming growth factor- $\beta 1$ (TGF- $\beta 1)$, mothers against decapentaplegic homolog 2 (Smad2), Smad3, and caspase-3 messenger ribonucleic acids (mRNAs) via RT-PCR. The total RNA was extracted from thoracic aorta tissues with TRIzol, and RT-PCR amplification was performed with RT-PCR kit (Thermo fisher, Waltham, MA, USA). Pre-denaturation at $94^{\circ} \mathrm{C}$ for $10 \mathrm{~min}$, denaturation at $94^{\circ} \mathrm{C}$ for $15 \mathrm{sec}$ and renaturation at $60^{\circ} \mathrm{C}$ for $60 \mathrm{sec}$ for 45 cycles. Primer sequences: Smad2-forward: 5'-CTTGACGCAGGGACTGTCCA-3' and Smad2-reverse: 5'-ACCTCTTTGAGCGCCACTAC-3', with the product length of 129bp,Smad3-forward:5'-CTTGGTGCAGAGACTGTCA-3' and Smad3-reverse: 5'-TTCTCTGTGATTGCCACTGC-3', with the product length of $129 \mathrm{bp}$, caspase-3-forward: 5'-CCAA CTGCAGACTGTCCAGA-3' and caspase-3-reverse: 5'-CAGG CTCCAGAAGAAGTTGG-3', TGF- $\beta 1$-forward: 5'-TGAGTG GCTGTCTTTTGACG-3' and TGF- $\beta 1$-reverse: 5'-ACTGAA GCGAAAGCCCTGTA-3', and $\beta$-actin-forward: 5'-GTCAGG TCATCACTATCGGCAAT-3' and $\beta$-actin-reverse: 5'-AGAG GTCTTTACGGATGTCAACGT-3'.

Detection of the expression levels of TGF- $\beta 1$, Smad2, Smad3 and caspase -3 proteins via western blot analysis. Thoracic aorta tissues were cut up with scissors, and the total protein was extracted by using pre-cooled tissue lysates. Bradford 

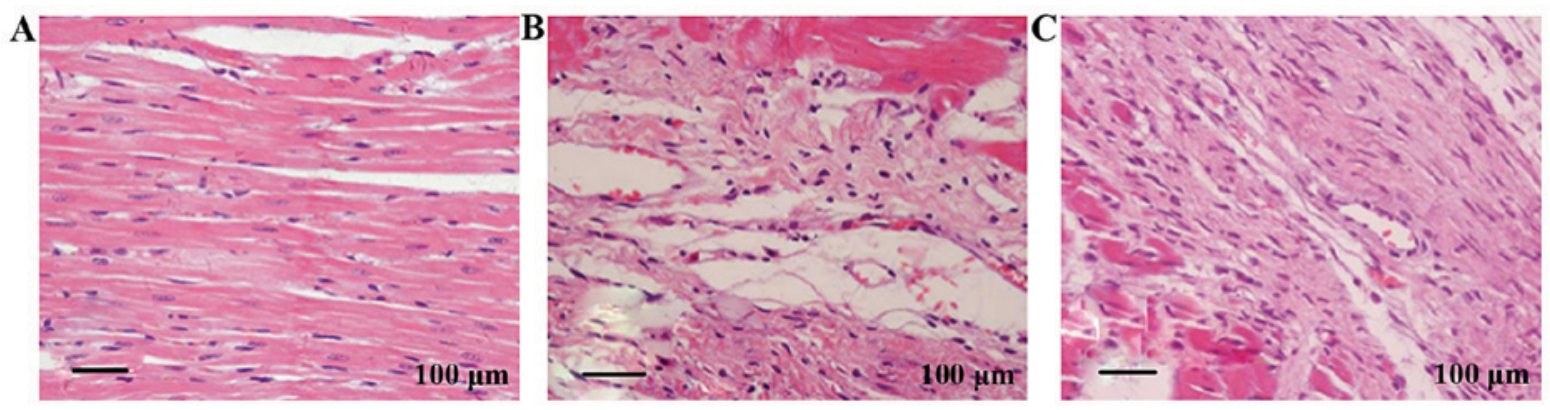

Figure 1. Hematoxylin and eosin (H\&E) staining results of myocardial tissues in each group of rats. (A) Sham operation group, (B) model group, (C) Qishen Yiqi dropping pill treatment group.

Table I. Comparison of the expression levels of TGF- $\beta 1$, Smad2, Smad3 and caspase-3 mRNAs at different time points in each group of rats (mean $\pm \mathrm{SD}, \mathrm{n}=5)$.

\begin{tabular}{lcccc}
\hline Groups & TGF- $\beta 1$ & Smad2 & Smad3 & Caspase-3 \\
\hline Sham operation & $1.00 \pm 0.00$ & $1.00 \pm 0.00$ & $1.00 \pm 0.00$ & $1.00 \pm 0.00$ \\
Model & $6.23 \pm 1.28^{\mathrm{a}}$ & $7.25 \pm 1.22^{\mathrm{a}}$ & $7.87 \pm 1.44^{\mathrm{a}}$ & $5.33 \pm 0.85^{\mathrm{a}}$ \\
Qishen Yiqi dropping pill treatment & $2.53 \pm 0.85^{\mathrm{a}, \mathrm{b}}$ & $4.60 \pm 0.62^{\mathrm{a}, \mathrm{b}}$ & $4.46 \pm 0.55^{\mathrm{a}, \mathrm{b}}$ & $2.85 \pm 0.85^{\mathrm{a}, \mathrm{b}}$ \\
\hline
\end{tabular}

${ }^{\text {a }}<0.05$ vs. sham operation group; ${ }^{\text {}} \mathrm{P}<0.05$ vs. model group.

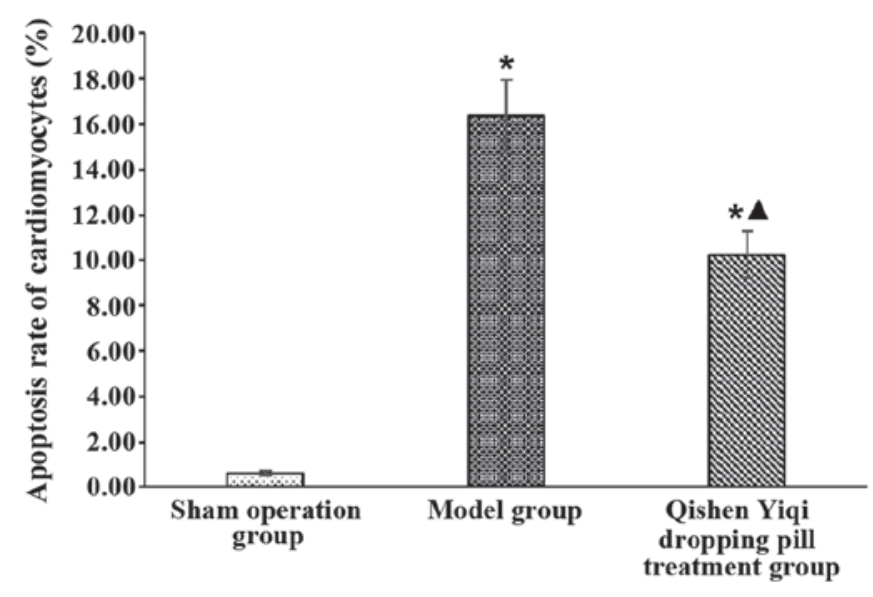

Figure 2. Comparison of the apoptosis rate of cardiomyocytes in each group of rats $\left({ }^{*} \mathrm{P}<0.05\right.$ vs. sham operation group, ${ }^{\wedge} \mathrm{P}<0.05$ vs. model group).

assay was applied to determine the protein content of samples, and $12 \%$ gels were used for protein separation. Proteins on the gels were transferred onto a polyvinylidene fluoride (PVDF) membrane by using membrane transfer equipment (wet transfer) at $100 \mathrm{~V}$ for $1.5 \mathrm{~h}$ and blocked with skim milk powder for $2 \mathrm{~h}$. After membrane washing, the proteins bound to TGF- $\beta 1, \mathrm{Smad} 2, \mathrm{Smad} 3$ and caspase-3 monoclonal antibodies $(1: 1,000)$ overnight, followed by color development using DAB. The gel imaging and chemiluminescence analysis system were employed to collect chromogenic bands. The chemiluminescence analysis kit was purchased from Shanghai Xin Yu Biological Technology Co., Ltd. (Shanghai, China). Quantity One software (Bio-Rad Laboratories, Inc., Hercules, CA, USA) was applied for protein band data analysis.
Statistical analysis. In statistical analysis, all data were analyzed and processed by using SPSS 18.0 (SPSS, Inc., Chicago, IL, USA). The $\chi^{2}$ test was conducted for enumeration data. Measurement data are expressed as (mean \pm SD), and the t-test was performed at the same time. $\mathrm{P}<0.05$ was considered to indicate a statistically significant difference.

\section{Results}

Histopathological results. In the sham operation group, cardiomyocytes were stained evenly and arranged neatly and densely with clear structures. In the model group, the cell morphology was fuzzy, the myocytes were hypertrophied, the nuclear pyknosis was fragmented, the arrangement was disordered, the intercellular space was narrowed, and the cytoplasm was missing. In the Qishen Yiqi dropping pill treatment group, the cell morphology tended to be normal (Fig. 1).

Cell apoptosis degree. The apoptotic rates of cardiomyocytes in the sham operation, the model and the Qishen Yiqi dropping pill treatment group were $0.68 \pm 0.22 \%, 16.35 \pm 3.58 \%$ and $10.25 \pm 2.28 \%$, respectively. The apoptosis rates of cardiomyocytes in the model group and the Qishen Yiqi dropping pill treatment were significantly higher than that in the sham operation group $(\mathrm{P}<0.05)$, and there was a significant difference in the comparison between the model and the Qishen Yiqi dropping pill treatment group $(\mathrm{P}<0.05)$ (Fig. 2).

Myocardial infarction area. The apoptosis rates of cardiomyocytes in the sham operation, the model, and the Qishen Yiqi dropping pill treatment group were $0.72 \pm 0.25 \%$, $38.56 \pm 6.89 \%$ and $22.15 \pm 5.15 \%$, respectively. The myocardial infarction areas in the model group and the Qishen Yiqi 
Table II. Comparison of the expression levels of TGF- $\beta 1, \operatorname{Smad} 2, \operatorname{Smad} 3$ and caspase- 3 proteins at different time points in each group of rats (mean $\pm \mathrm{SD}, \mathrm{n}=5)$.

\begin{tabular}{lcccc}
\hline Groups & TGF- $\beta 1$ & Smad2 & Smad3 & Caspase-3 \\
\hline Sham operation & $1.00 \pm 0.00$ & $1.00 \pm 0.00$ & $1.00 \pm 0.00$ & $1.00 \pm 0.00$ \\
Model & $5.11 \pm 0.83^{\mathrm{a}}$ & $4.39 \pm 0.83^{\mathrm{a}}$ & $5.89 \pm 0.35^{\mathrm{a}}$ & $2.83 \pm 0.51^{\mathrm{a}}$ \\
Qishen Yiqi dropping pill treatment & $3.51 \pm 0.53^{\mathrm{a}, \mathrm{b}}$ & $3.29 \pm 0.75^{\mathrm{a}, \mathrm{b}}$ & $3.58 \pm 0.55^{\mathrm{a}, \mathrm{b}}$ & $1.80 \pm 0.26^{\mathrm{a}, \mathrm{b}}$ \\
\hline
\end{tabular}

${ }^{\mathrm{a}} \mathrm{P}<0.05$ vs. sham operation group; ${ }^{\mathrm{b}} \mathrm{P}<0.05$ vs. model group.

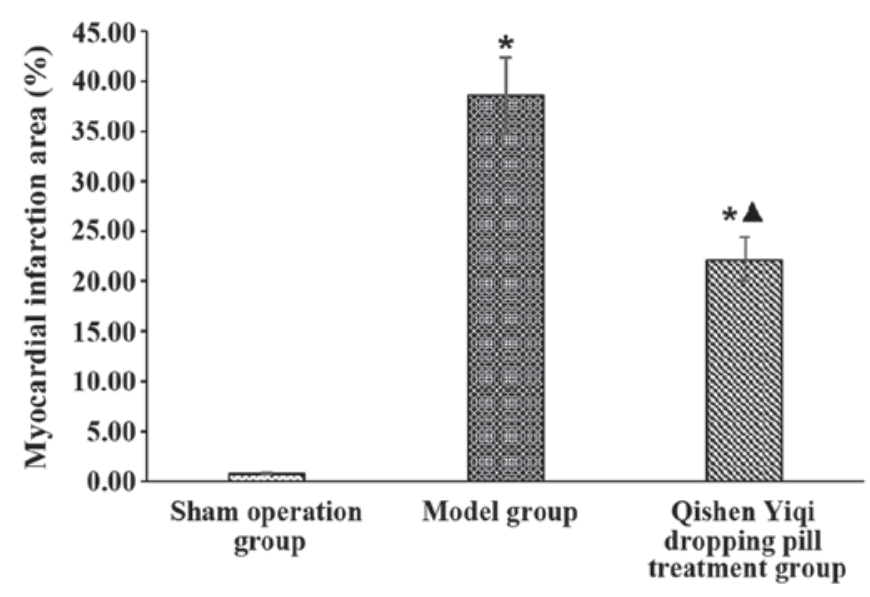

Figure 3. Comparison of the myocardial infarction area in each group of rats ${ }^{*} \mathrm{P}<0.05$ vs. sham operation group; ${ }^{\wedge} \mathrm{P}<0.05$ vs. model group.

dropping pill treatment were remarkably larger than that in the sham operation group $(\mathrm{P}<0.05)$, and there was an obvious difference in the comparison between the model and the Qishen Yiqi dropping pill treatment group $(\mathrm{P}<0.05)$ (Fig. 3).

RT-PCR and western blot analysis detection results. The expression levels of TGF- $\beta 1, \operatorname{Smad} 2, \operatorname{Smad} 3$, and caspase-3 mRNAs and proteins in the model group and the Qishen Yiqi dropping pill treatment group were obviously higher than those in the sham operation group $(\mathrm{P}<0.05)$, and there were significant differences in the comparison between the model and the Qishen Yiqi dropping pill treatment group $(\mathrm{P}<0.05)$ (Tables I and II).

\section{Discussion}

Chronic heart failure (CHF) is a complex clinical syndrome of ventricular ejection or filling disorders due to structural or functional abnormalities in the heart. CHF not only has a high prevalence rate but also has a poor prognosis, and it has become one of the leading causes of death among the elderly $(4,5)$. Most scholars agree that the pathological remodeling of the left ventricle is the most important pathophysiological mechanism of the occurrence of CHF. Decreased cardiac function and left ventricular hypertrophy are the main signs of left ventricular remodeling. In current clinical treatment for $\mathrm{CHF}$, $\beta$-blockers and angiotensin-converting enzyme inhibitors are used to inhibit neuroendocrine activation during heart failure generally based on the symptomatic treatments such as vasodilation, cardiac arrest and diuresis so as to delay and prevent myocardial remodeling, thereby reducing the patient's hospitalization and mortality rates (6,7). However, in general, the overall efficacy of western medicine in the treatment of $\mathrm{CHF}$ is unsatisfactory.

The treatment of $\mathrm{CHF}$ with traditional Chinese medicine has a long history, which can alleviate the symptoms of patients, improve their quality of life, and delay the occurrence and development of CHF. In the theory of Traditional Chinese medicine, CHF has been included in the category of syndromes such as 'edema', 'thoracic obstruction', 'dyspnea with cough' and 'palpitation'. It is believed that due to multiple etiologies, the body's qi, blood, yin and yang are impaired. Zang-Fu imbalance leads to internal retention of water with extravasated blood. The pathogenesis of the disease is blood stasis, the heart-yang, water-rheum collecting internally and heart-qi deficiency, and its treatment is based on the principles of promoting blood circulation and collaterals and replenishing yang. Qishen Yiqi dropping pills are a traditional Chinese medicine preparation composed of Danshen, Huangqi, Jiangxiang and Sanqi, which have the functions of activating blood circulation, relieving pain and promoting circulation of $q i$. With a stable dosage form and reliable clinical efficacy, it is one of the representative drugs in the treatment of heart disease with traditional Chinese medicine (8-10).

Previous studies have revealed that Qishen Yiqi dropping pills play roles in expanding coronary blood vessels, increasing coronary sinus blood oxygen content and coronary blood flow volume, improving myocardial blood and oxygen supply, reducing myocardial oxygen consumption index, elevating cardiac stroke output and cardiac output, increasing the maximum rate of increase of left ventricular pressure, and adjusting cardiac compliance $(11,12)$. At the same time, Qishen Yiqi dropping pills can lower the platelet aggregation rate, reduce the thickness of aortic plaques, tend to reduce the area of aortic plaques, and have the effects of preventing and treating atherosclerosis and anti-lipid peroxidation (13). The myocardial protective effect of Qishen Yiqi dropping pills on CHF rats was investigated in this study. The results indicated that after treatment with Qishen Yiqi dropping pills, the morphology of myocardial cells tended to be normal, and the rate of apoptosis and myocardial infarction area were decreased, suggesting that Qishen Yiqi dropping pills can protect the myocardium, and its possible mechanism is to reduce the degree of myocardial cell apoptosis and inhibit the fibrosis of myocardial cells. 
Increased expression level of TGF- $\beta 1$ in the vascular wall can promote vascular smooth muscle cell proliferation, migration, extracellular matrix deposition, lipid accumulation in the arterial wall and inflammatory cell infiltration, and these factors are all crucial steps in myocardial cell fibrosis (14). As the main downstream mediators of TGF- $\beta 1$, Smad 2 and Smad 3 play important roles in the process of myocardial fibrosis (15). After phosphorylation, Smad2/3 can bind to Smad4 to form a complex involved in the regulation of gene transcription, so as to promote the expression of collagens as well as the formation and progression of myocardial fibrosis. Caspase- 3 is one of the important genes for apoptosis. The results of RT-PCR and western blot analysis in this study manifested that Qishen Yiqi dropping pills might improve the degree of myocardial fibrosis by suppressing the TGF- $\beta 1 /$ Smads pathway and inhibit apoptosis of cardiomyocytes by impeding the caspase- 3 signaling pathway, thus protecting the myocardium.

In summary, Qishen Yiqi dropping pills obviously protect the myocardium of CHF rats, which may improve the degree of myocardial fibrosis by impeding the TGF- $\beta 1 /$ Smads pathway and improve cardiomyocyte apoptosis by inhibiting the caspase-3 signaling pathway, so as to play a role in protecting the myocardium. However, since traditional Chinese medicine is characterized by multiple components, multiple targets and comprehensive treatments, the conclusions of this study have to be further verified.

\section{Acknowledgements}

Not applicable.

\section{Funding}

No funding was received.

\section{Availability of data and materials}

The datasets used and/or analyzed during the present study are available from the corresponding author on reasonable request.

\section{Authors' contributions}

YL and DW wrote the manuscript. YL, DW and XY established the rat model of CHF. MW and ZL helped with TUNEL. XB and $\mathrm{CZ}$ performed PCR. HJ was responsible for western blot analysis. All authors read and approved the final manuscript.

\section{Ethics approval and consent to participate}

The study was approved by the Ethics Committee of Zhengzhou Central Hospital Affiliated to Zhengzhou University (Zhengzhou, China).

\section{Patient consent for publication}

Not applicable.

\section{Competing inetrests}

The authors declare they have no competing interests.

\section{References}

1. Abraham WT, Stevenson LW, Bourge RC, Lindenfeld JA, Bauman JG and Adamson PB; CHAMPION Trial Study Group: Sustained efficacy of pulmonary artery pressure to guide adjustment of chronic heart failure therapy: Complete follow-up results from the CHAMPION randomised trial. Lancet 387: 453-461, 2016.

2. Sharma A, Lavie CJ, Borer JS, Vallakati A, Goel S, LopezJimenez F, Arbab-Zadeh A, Mukherjee D and Lazar JM: Meta-analysis of the relation of body mass index to all-cause and cardiovascular mortality and hospitalization in patients with chronic heart failure. Am J Cardiol 115: 1428-1434, 2015.

3. JianXin C, Xue X, ZhongFeng L, Kuo G, FeiLong Z, ZhiHong L, Xian W and HongCai S: Qishen Yiqi Drop Pill improves cardiac function after myocardial ischemia. Sci Rep 6: 24383, 2016.

4. Satake H, Fukuda K, Sakata Y, Miyata S, Nakano M, Kondo M, Hasebe Y, Segawa M and Shimokawa H; CHART-2 Investigators: Current status of primary prevention of sudden cardiac death with implantable cardioverter defibrillator in patients with chronic heart failure - A report from the CHART-2 Study. Circ J 79: 381-390, 2015.

5. Troughton RW, Frampton CM, Brunner-La Rocca HP, Pfisterer M, Eurlings LW, Erntell H, Persson H, O'Connor CM, Moertl D, Karlström P, et al: Effect of B-type natriuretic peptide-guided treatment of chronic heart failure on total mortality and hospitalization: An individual patient meta-analysis. Eur Heart J 35: 1559-1567, 2014.

6. Gaggin HK, Szymonifka J, Bhardwaj A, Belcher A, De Berardinis B, Motiwala S, Wang TJ and Januzzi JL Jr: Headto-head comparison of serial soluble ST2, growth differentiation factor-15, and highly-sensitive troponin $\mathrm{T}$ measurements in patients with chronic heart failure. JACC Heart Fail 2: 65-72, 2014.

7. Arzt M, Woehrle H, Oldenburg O, Graml A, Suling A, Erdmann E, Teschler $\mathrm{H}$ and Wegscheider K; SchlaHF Investigators: prevalence and predictors of sleep-disordered breathing in patients with stable chronic heart failure: The SchlaHF Registry. JACC Heart Fail 4: 116-125, 2016.

8. Yang Q and Cao Y: Study on mechanisms and myocardial protective effect of Qishen Yiqi dropping pills on rats with myocardial infarction. Zhonghua Wei Zhong Bing Ji Jiu Yi Xue 29: 501-505, 2017 (In Chinese).

9. Yu FC, Xu YJ, Tong JY, Lu ZZ and Zhang XH: Therapeutic effects of Qishen Yiqi Dropping Pill on myocardial injury induced by chronic hypoxia in rats. Chin J Nat Med 13: 776-780, 2015.

10. Cui ZT, Wei WL, Liu M and Wang WJ: Effect of pretreatment with qishen yiqi dropping pills on right cardiac function of patients undergoing valve replacement. Zhongguo Zhong Yao Za Zhi 39: 916-919, 2014 (In Chinese).

11. Liu W, Gao FF, Li Q, Lv JW, Wang Y, Hu PC, Xiang QM and Wei L: Protective effect of astragalus polysaccharides on liver injury induced by several different chemotherapeutics in mice. Asian Pac J Cancer Prev 15: 10413-10420, 2014.

12. Wang J, Lu L, Wang Y, Wu Y, Han J, Wang W, Li C and Tu P: Qishenyiqi Dropping Pill attenuates myocardial fibrosis in rats by inhibiting RAAS-mediated arachidonic acid inflammation. J Ethnopharmacol 176: 375-384, 2015.

13. Wang Y, Wang Q, Li C, Lu L, Zhang Q, Zhu R and Wang W: A review of Chinese herbal medicine for the treatment of chronic heart failure. Curr Pharm Des 23: 5115-5124, 2017.

14. Zhao M, Zheng S, Yang J, Wu Y, Ren Y, Kong X, Li W and Xuan J: Suppression of TGF- $\beta 1 /$ Smad signaling pathway by sesamin contributes to the attenuation of myocardial fibrosis in spontaneously hypertensive rats. PLoS One 10: e0121312, 2015.

15. Li S, Fan Q, He S, Tang T, Liao Y and Xie J: MicroRNA-21 negatively regulates Treg cells through a TGF- $\beta 1 /$ Smad-independent pathway in patients with coronary heart disease. Cell Physiol Biochem 37: 866-878, 2015.

This work is licensed under a Creative Commons Attribution-NonCommercial-NoDerivatives 4.0 International (CC BY-NC-ND 4.0) License. 